\title{
Analytical Procedure Robustness
}

National Cancer Institute

\section{Source}

National Cancer Institute. Analytical Procedure Robustness. NCI Thesaurus. Code C134245.

The measure of a procedure's capacity to remain unaffected by small, but deliberate variations in method parameters and provides an indication of its reliability during normal usage. 